\title{
Using feedback tools to enhance the quality and experience of care
}

\author{
Jan R. Boehnke ${ }^{1}$. Claudia Rutherford ${ }^{2,3}$ \\ Published online: 11 October 2021 \\ (C) The Author(s), under exclusive licence to Springer Nature Switzerland AG 2021
}

\section{Introduction}

In 2019, we issued a call for papers interested in approaches to data-based feedback mechanisms to enhance quality and experience of care, focusing on (health-related) quality of life (HRQL). We were looking for current and innovative state-of-the-art use of data-based feedback mechanisms to enhance quality and experience of care. For the purposes of this special issue, we defined 'data-based feedback mechanisms' as systems that generate and capture data during an individual care process and that are fed back into the same individual care process. In line with the scope of our journal covering quality of life aspects of treatment, care, and rehabilitation, we kept 'care process' intentionally broad, describing active treatments in inpatient and outpatient settings, as well as supportive care and other interventions extending beyond typical treatment settings. We received the exceptional number of 29 expressions of interest of which 22 papers are now collected in this special issue. For the issue, we have grouped the studies in four sections that align with elements of the call. But as all of the studies were deeply rooted in clinical practice and applications, there are a lot of themes that are common across the studies and sections, and we invite readers to explore and cross-read these papers with their own research and practice in mind.

Jan R. Boehnke

j.r.boehnke@dundee.ac.uk

1 School of Health Sciences, University of Dundee, City Campus, 11 Airlie Place, Dundee DD1 4HJ, UK

2 Faculty of Science, School of Psychology, Quality of Life Office, The University of Sydney, Sydney, Australia

3 Faculty of Medicine and Health, Susan Wakil School of Nursing and Midwifery, Cancer Nursing Research Unit (CNRU), The University of Sydney, Sydney, Australia

\section{The special issue}

The issue opens with a focus on implementation in clinical practice. It has been ten years since the "User's Guide for Implementing Patient-Reported Outcomes Assessment in Clinical Practice" [1] was published by the International Society for Quality of Life Research (ISOQOL). It was motivated by the potential for Patient-Reported Outcomes (PROs) to enhance patient-centered care and the lack of resources describing options, steps involved in planning as well as a discussion of disadvantages of and alternatives to their use. But although the "User's Guide" is now in its second edition with a companion guide and first translations, systematic approaches to implementing systems and their evaluation remain rare. A recent review of implementation in cancer care suggests that reports of real-world PRO implementations are limited [2] and especially connections with, and emphasis on, implementation science frameworks remains limited [3-5]. The first set of five papers of the special issue were submitted on behalf of the 'ISOQOL PROMs/PREMs in Clinical Practice Implementation Science Work Group' [4, 6-9] and describe how implementation science theories and frameworks can guide translation processes from research to practice. The papers are based on a set of case studies describing the use of five well-established implementation science frameworks (for an overview refer to Table 1 in [4]), illustrating how multidisciplinary approaches support implementation in practice settings and increase the likelihood of adoption.

The second set of papers presents system perspectives at very different stages of development. Mental health services are a clinical area where the use of PROs is fairly established [10, 11]. Three papers report the processes at the beginning of a system development by an international collaboration, developing a clinical feedback system for a practice-research network in Norway [12]. The first paper [13] reports the stages involved in the development of the tool, offering insights into a continuous quality improvement perspective and it stresses the key dimension of engaging with stakeholders. The accompanying paper [14] reports 
early evaluation results for the underlying patient-reported outcome measures' (PROMs) psychometric properties and it highlights opportunities arising from engagement with the target population for this process. The third paper [15] focuses on the question of how continuous feedback systems are perceived by patients-an evaluation that forms a critical part of any implementation process, but there is still relatively little systematic evidence available as the authors argue. The results reinforce the observation that PROMs are communicative tools and their use is a contextspecific intentional process, a perspective that informs the implementation, use, and research on PROMs increasingly [16-20]. The fourth paper [21] describes the development of a smartphone app for monitoring asthma in children and adolescents in seven distinct phases from conceptualization to usability evaluation. The paper presents a substantial amount of process learning and especially the discussion of technical and legal issues that the project faced are a unique contribution within this special issue, which highlights important operational aspects to consider when developing such systems. The fifth paper [22] illustrates the contribution of such systems from a different point of the life span of such research and practice programs. Using the concept of "Standardized assessment, information, and networking technologies" (SAINTs), the authors summarize findings from their practice-research network spanning nearly three decades of development. Focusing on working with patients with chronic conditions, the authors present findings and strategies that potentially improve patient health and satisfaction. In addition to a focus on what makes SAINTs easier to use and implement, their experience stresses the importance of (i) technology serving patients and (ii) engaging them and their clinicians in the co-production of better care. The section concludes with a paper [23] that stretches the system-level view in another direction as it summarizes the first steps of developing and implementing an electronic platform to collect PROMs and patient-reported experience measures (PREMs) from all secondary care patients across Wales, UK. The processes involved to reach the launch of a fully integrated national electronic data collection platform (in 2016 including over 30 measures) are presented and accompanied with lessons learned including a map of future research and implementation goals.

Papers in the sections on implementation in clinical practice as well as on system perspectives consistently emphasize how important the underlying technical processes and systems are. The third section focuses on underlying principles and technologies. The first paper [24] describes how predictive analytics were integrated into a clinical feedback system to facilitate shared decision reports for patients with advanced knee and hip osteoarthritis. The paper summarizes multi-stage and mixed methods research and especially focuses on the integration of an existing registry to support this system efficiently with relevant reference data. One of the most challenging aspects for the development of feedback systems is the provision of "individualized shared decision reports." While usually built on nomothetic data, they are used to make predictions about ideographic developments and it is by no means clear, whether these apply to a particular patient [25-27]. The paper on the "ESMvis" tool [28] presents a new analysis framework using experience sampling data for descriptive feedback, focusing on direct visualization of the dynamic nature of raw data of a single individual. The paper is accompanied by a range of resources including R [29] and shiny [30] visualization tools to produce personalized reports with high relevance to the individual care process. As discussed by the authors, the use of mobile devices provides rich idiographic information, but more work is required to develop theoretical and professional frameworks to include it in care processes. Open-source applications with high utility are a way to facilitate exploration of how to embed this technology in professional practice. The use of open-source software to explore data on an ongoing basis is also the topic of the third paper in this section [31]. The team focuses on how to implement dashboards for tracking data return and various other study data in a trial environment—-but the capability likely extends to practice applications that also depend on quality control techniques to improve response rates. Sharing of such approaches, including the underlying syntax and workflows, can help the field develop (common) standards and lower hurdles for implementation in practice settings. The final paper in this section [32] used the Statistical Analysis System (SAS) software to develop a dashboard collecting relevant health status information from participants and to support them to engage with this data. Embedded in theoretical frameworks such as Knowledge-to-Action ([33]; see Fig. 1 in [32]) and Wilson \& Cleary's outcome model [34], the paper presents processes and syntax shared on the Open Science Framework (https://osf.io/3gzcx/).

The issue closes with a section on practice-based applications. Four of these papers are drawn from different cancer care settings and explore the experience of users with feedback systems as well as the feasibility of and considerations for implementation. For example, moving the collection of PROMs to electronic formats still offers a variety of options and their uptake, experience, and accessibility may be influenced by factors in the target population. Samuel et al. [35] investigate the question of how far the adoption of health information technologies varies along social gradients and describe differences associated with ethnicity in bladder and prostate cancer patients. The two main themes identified in the second paper [36] offer additional insight into the potential of electronic PRO (ePRO) systems when supporting post-hospital or outpatient care: the system was described as providing "reassurance" and "empowerment" 
(Table 3 in [36]), pointing to a range of functions such a system could fulfill in such a setting. A report investigating the implementation of a monthly text-based symptommonitoring program found such a system feasible, with good adherence rates, and was positively perceived by patients and staff [37]. Nevertheless, patients who were more aware of their symptom levels and less hesitant to engage with their health care providers found the questions too similar to assessments during treatment visits. The QOLIBRY study [38] investigated the feasibility of using an ePRO in clinical settings in France and found differences in the use across physicians included in the study-familiarity with the systems, usefulness of the feedback, and perceived appropriateness for the treatment process are discussed as reasons by the authors. Leaving cancer care, the online evaluation of the KLIK PROM portal through clinicians using this system [39] mirrors a number of these topics in pediatric care; and the sixth paper in this section [40] investigates general practitioners' views about depression screening combined with targeted feedback. These studies illustrate that patients place value on information sharing and potentially also improved communication with their clinicians; but clinicians need to be open to using these systems, systems need to connect to their needs in the specific consultation context, and clinicians need to be trained in their use [41]. The special issue closes with a paper [42] reporting secondary analyses of a trial investigating the effectiveness of routine outcome monitoring in a mental health outpatient service offering cognitive-behavioral therapy. In addition to a detailed description of how session-by-session PROMs data is collected [43], the analyses suggest that in this setting session number, suicidality, therapy motivation, and the occurrence of life events were predictive of deterioration in the later therapy sessions.

\section{Editorial commentary}

One could wonder about the topic of such a special issue, considering that in recent decades, a substantial amount of effort in our field has focused not only on measuring HRQL as an outcome in efficacy and effectiveness studies, but also for use as a treatment target [44]. Feedback systems offer a way to translate nomothetic efficacy and effectiveness research into individual care processes and to evaluate whether the current treatment works for a particular patient $[10,45]$. Different disciplines of health research and practice influenced by geography, legal frameworks, and requirements of health care systems adopted use of PROMs at different speeds. We therefore felt that a call for papers reporting ongoing research and practice highlights the continued importance of the topic. The breadth of papers shows that it is a lively research and practice field that is valued by several stakeholders, including and potentially foremost patients. But widening the perspective, challenges remain for the use of feedback systems. It is beyond the scope of this editorial to provide a review of the relevant research, practice, and professional fields informing the use of feedback systems. But we take the opportunity to stress the following five perspectives that struck us as particularly relevant when reflecting on this special issue.

First, it is important to mention for balance that, despite at least thirty years of formal models, concepts, and research, the use of feedback systems (and especially the use of PROMs for feedback purposes) remains a contested area. Conclusions of reviews of the effects of systematically providing feedback based on PROMs into ongoing care processes vary widely. Early reviews suggest that there may be effects on the quality of care processes (e.g., better diagnosis and assessment [46]), but that the effect on functional outcomes may be limited ([47, 48]; for a narrative review of earlier evidence see [49]). More recent reviews differ for example in their conclusions by field of application, as there are potential effects in mental health (see for example, [11] for an exhaustive list of previous meta-analyses in the area and [50] for an opposing systematic review), but less likely in other health domains (for example [51, 52]); or whether the studies investigate an effect of providing feedback that should manifest in all patients and care processes (potentially not [50-52]) or rather an effect on care process of patients who deteriorate or are at risk of deterioration (potentially yes, but mainly investigated in mental health contexts [11]). And the individual papers of this special issue provide additional sources of supporting evidence or discussion of the evidence base for feedback use in their respective fields. Due to the divergence of findings and the difficulty to disentangle particular service contexts, geographies, and underlying philosophies of what a feedback system should deliver, the question whether feedback systems generally improve outcomes (for all or particular subgroups of patients) remains unanswered. Reviews of studies in the area also typically find that improvements in methodology are required to provide a more robust evidence base [49-53]. Therefore, this remains a key area of primary and meta-research and a responsibility to provide evidence of improved outcomes for any implementation, if this is the goal of the feedback system.

Second, while improving individual outcomes is a possible motivation for implementing feedback systems, another one is to support patient-clinician communication and patient-centered care more broadly [1, 49, 54]. A number of the papers of the special issue speak to this dimension and future directions of research for this area are discussed elsewhere [16, 55]. Further, research and practice are in this regard at least equally diverse as with view to outcomes: what does communication mean, how does 
patient-centeredness manifest, and what are good ways to demonstrate that feedback tools increase or improve this aspect of care, remain important areas of research.

Third, these two burning issues are tightly connected to the question of the theoretical foundations of this field of research and professional practice. It has been highlighted in the past that there is a wide range of theoretical frameworks inside and outside the PRO-interested community that can address many relevant aspects of how feedback systems (are supposed to) work [3, 56, 57]. Without strong theoretical frameworks unifying existing evidence as well as guiding confirmatory research as part of a multi-method research agenda, fundamental questions about the mechanisms and usefulness of feedback systems will remain unanswered.

Fourth, it is imperative to remember the context in which feedback systems are used and the ethical questions that are raised by it. "It is a truth universally acknowledged" [58] that in health care settings, a wide range of data are collected on each patient and several of the papers describe the range of data sources available to stakeholders. In most cases, narratives on organizational or between-organizational level support the collection of these data, but only more recently has PRO data been connected to these and resulting synergies are explored more actively [59-61]. But within this context, PRO data only add to the detail held by services, which raises operational and ethical questions about privacy, ownership, and access [62,63]. Strong theories about the use of feedback systems as well as a robust evidence base (either generally or for the respective application) would provide key points of reference in these debates. And as PRO data will in the overwhelming number of cases be part of larger data capture and management systems, a question is how theories about feedback systems fit in or link to theories about clinical data management, provision, and use more broadly. A second ethical question has been raised more recently, from the perspective of feedback systems as health technologies and parts of an evidence-based approach to care provision. If feedback systems improve the quality of care processes in this sense, does this generate an ethical imperative to use them (as for example recently argued by [64])? And if they are used, what do stakeholders accessing the feedback actually do? The connection between evidence base, evidence-based practice, and professions engaging with feedback systems becomes here particularly pertinent.

Fifth, creating better understanding regarding how to implement such systems and what implementation actually means is an important perspective to which a number of papers, and especially the first section, make a strong contribution and invite more studies with detailed reporting of systems and system development processes [2]. But this also includes a very concrete and technical level, i.e., the question of how such systems work and how they work optimally. Several papers in this issue and especially the section on principles and technologies provide some insight into the "workings under the hood." But it needs to be acknowledged that the technical and technological problems for implementing such systems are important barriers [65], including how to present the generated information [66]. Another point related to implementation of the systems is the question of once a system is implemented, how is it developed, maintained, and sustainably retained as part of service structures, and which organizational narratives and mechanisms support this $[55,57,59]$ ?

The call for papers was not geared towards answering these questions, but rather to elicit current and state-of-theart research and practice focusing on such systems. We are grateful for the excellent range of submissions received and to all authors and reviewers involved in selecting the published papers. These issues raised in our commentary are only a small selection relevant to us as an editorial team and to the whole research field. But many other issues raised by the papers are less specific to the use of feedback systems and closely related to the remit of Quality of Life Research, such as the development and evaluation of the PROMs used within these systems; considerations of equity of access to technologies and investigating equity of outcomes; or what matters to patients in care for their conditions.

\section{References}

1. Snyder, C. F., Aaronson, N. K., Choucair, A. K., Elliott, T. E., Greenhalgh, J., Halyard, M. Y., et al. (2012). Implementing patient-reported outcomes assessment in clinical practice: a review of the options and considerations. Quality of Life Research, 21(8), 1305-1314. https://doi.org/10.1007/s11136-011-0054-x

2. Anatchkova, M., Donelson, S. M., Skalicky, A. M., McHorney, C. A., Jagun, D., \& Whiteley, J. (2018). Exploring the implementation of patient-reported outcome measures in cancer care: need for more real-world evidence results in the peer reviewed literature. Journal of Patient-Reported Outcomes, 2(1), 64. https://doi.org/ 10.1186/s41687-018-0091-0

3. de Jong, K. (2016). Deriving implementation strategies for outcome monitoring feedback from theory, research and practice. Administration and Policy in Mental Health, 43(3), 292-296. https://doi.org/10.1007/s10488-014-0589-6

4. Stover, A. M., Haverman, L., van Oers, H. A., Greenhalgh, J., Potter, C. M., \& Group, I. P. P. i. C. P. I. S. W. (2020). Using an implementation science approach to implement and evaluate patient-reported outcome measures (PROM) initiatives in routine care settings. Quality of Life Research. https://doi.org/10.1007/ s11136-020-02564-9

5. van der Wees, P. J., Verkerk, E. W., Verbiest, M. E. A., Zuidgeest, M., Bakker, C., Braspenning, J., et al. (2019). Development of a framework with tools to support the selection and implementation of patient-reported outcome measures. Journal of PatientReported Outcomes. https://doi.org/10.1186/s41687-019-0171-9

6. Manalili, K., Santana, M. J., \& group, I. P. P. i. c. p. i. s. w. . (2020). Using implementation science to inform the integration of electronic patient-reported experience measures (ePREMs) into healthcare quality improvement: description of a theory-based 
application in primary care. Quality of Life Research. https://doi. org/10.1007/s11136-020-02588-1

7. Ahmed, S., Zidarov, D., Eilayyan, O., \& Visca, R. (2020). Prospective application of implementation science theories and frameworks to inform use of PROMs in routine clinical care within an integrated pain network. Quality of Life Research. https://doi.org/10.1007/s11136-020-02600-8

8. van Oers, H. A., Teela, L., Schepers, S. A., Grootenhuis, M. A., Haverman, L., PROMs, I., , et al. (2020). A retrospective assessment of the KLIK PROM portal implementation using the consolidated framework for Implementation Research (CFIR). Quality of Life Research. https://doi.org/10.1007/s11136-020-02586-3

9. Roberts, N. A., Janda, M., Stover, A. M., Alexander, K. E., Wyld, D., Mudge, A., et al. (2020). The utility of the implementation science framework "integrated promoting action on research implementation in health services" (i-PARIHS) and the facilitator role for introducing patient-reported outcome measures (PROMs) in a medical oncology outpatient department. Quality of Life Research. https://doi.org/10.1007/s11136-020-02669-1

10. Howard, K. I., Moras, K., Brill, B. L., Martinovich, Z., \& Lutz, W. (1996). Evaluation of psychotherapy. Efficacy, effectiveness and patient progress. American Psychologist, 51, 1059-1064.

11. Lambert, M. J., Whipple, J. L., \& Kleinstäuber, M. (2018). Collecting and delivering progress feedback: a meta-analysis of routine outcome monitoring. Psychotherapy, 55(4), 520-537. https:// doi.org/10.1037/pst0000167

12. Castonguay, L. G., Barkham, M., Lutz, W., \& McAleavey, A. (2013). Practice-oriented research: Approaches and applications. In M. J. Lambert (Ed.), Bergin and Garfield's handbook of psychotherapy and behavior change (6th edition ed., pp. 85-133). Hoboken, NJ: John Wiley \& Sons.

13. Nordberg, S. S., McAleavey, A. A., \& Moltu, C. (2021). Continuous quality improvement in measure development: lessons from building a novel clinical feedback system. Quality of Life Research. https://doi.org/10.1007/s11136-021-02768-7

14. McAleavey, A. A., Nordberg, S. S., \& Moltu, C. (2021). Initial quantitative development of the norse feedback system: a novel clinical feedback system for routine mental healthcare. Quality of Life Research. https://doi.org/10.1007/s11136-021-02825-1

15. Solstad, S. M., Kleiven, G. S., \& Moltu, C. (2020). Complexity and potentials of clinical feedback in mental health: an in-depth study of patient processes. Quality of Life Research. https://doi. org/10.1007/s11136-020-02550-1

16. Greenhalgh, J., Gooding, K., Gibbons, E., Dalkin, S., Wright, J., Valderas, J., et al. (2018). How do patient reported outcome measures (PROMs) support clinician-patient communication and patient care? A realist synthesis. Journal of Patient-Reported Outcomes, 2, 42. https://doi.org/10.1186/s41687-018-0061-6

17. Kwon, J. Y., Thorne, S., \& Sawatzky, R. (2019). Interpretation and use of patient-reported outcome measures through a philosophical lens. Quality of Life Research, 28(3), 629-636. https://doi.org/10. 1007/s11136-018-2051-9

18. Truijens, F. L., Van Nieuwenhove, K., De Smet, M. M., Desmet, M., \& Meganck, R. (2021). How questionnaires shape experienced symptoms. A qualitative case comparison study of questionnaire administration in psychotherapy research. Qualitative Research in Psychology. https://doi.org/10.1080/14780887.2021. 1886383

19. Truijens, F. L., Desmet, M., De Coster, E., Uyttenhove, H., Deeren, B., \& Meganck, R. (2019). When quantitative measures become a qualitative storybook: a phenomenological case analysis of validity and performativity of questionnaire administration in psychotherapy research. Qualitative Research in Psychology. https://doi.org/10.1080/14780887.2019.1579287
20. McClimans, L. (2011). Interpretability, validity, and the minimum important difference. Theoretical Medicine and Bioethics, 32(6), 389-401. https://doi.org/10.1007/s11017-011-9186-9

21. Mayoral, K., Garin, O., Caballero-Rabasco, M. A., Praena-Crespo, M., Bercedo, A., Hernandez, G., et al. (2021). Smartphone app for monitoring asthma in children and adolescents. Quality of Life Research. https://doi.org/10.1007/s11136-020-02706-z

22. Wasson, J. H. (2020). Standardized assessment, information, and networking technologies (SAINTs): lessons from three decades of development and testing. Quality of Life Research. https://doi. org/10.1007/s11136-020-02528-z

23. Withers, K., Palmer, R., Lewis, S., \& Carolan-Rees, G. (2020). First steps in PROMs and PREMs collection in Wales as part of the prudent and value-based healthcare agenda. Quality of Life Research. https://doi.org/10.1007/s11136-020-02711-2

24. Franklin, P. D., Zheng, H., Bond, C., \& Lavallee, D. C. (2020). Translating clinical and patient-reported data to tailored shared decision reports with predictive analytics for knee and hip arthritis. Quality of Life Research. https://doi.org/10.1007/ s11136-020-02557-8

25. Krause, M. S., Lutz, W., \& Boehnke, J. R. (2011). The role of sampling in clinical trial design. Psychotherapy Research, 21(3), 243-251.

26. Fisher, A. J., Medaglia, J. D., \& Jeronimus, B. F. (2018). Lack of group-to-individual generalizability is a threat to human subjects research. Proceedings of the National Academy of Sciences, 115(27), E6106-E6115. https://doi.org/10.1073/pnas.1711978115

27. Molenaar, P., \& Campbell, C. G. (2009). The new person-specific paradigm in psychology. Current Directions in Psychological Science, 18(2), 112-117.

28. Bringmann, L. F., van der Veen, D. C., Wichers, M., Riese, H., \& Stulp, G. (2020). ESMvis: a tool for visualizing individual experience sampling method (ESM) data. Quality of Life Research. https://doi.org/10.1007/s11136-020-02701-4

29. R Core Team (2021). R: A language and environment for statistical computing. (4.1.1 ed.). Vienna, Austria: R Foundation for Statistical Computing.

30. Chang, W., Cheng, J., Allaire, J., Sievert, C., Schloerke, B., Xie, Y., et al. (2021). shiny: Web Application Framework for R. (1.7.0 ed.).

31. Golafshar, M. A., Petersen, M., Vargas, C. E., Samadder, N. J., Kunze, K. L., McCormick, N., et al. (2020). Utilizing open-source platforms to build and deploy interactive patient-reported quality of life tracking tools for monitoring protocol adherence. Quality of Life Research. https://doi.org/10.1007/s11136-020-02617-z

32. Mozafarinia, M., Rajabiyazdi, F., Brouillette, M. J., Fellows, L. K., \& Mayo, N. E. (2020). Development and usability of a feedback tool, "my personal brain health dashboard", to improve setting of self-management goals among people living with HIV in Canada. Quality of Life Research. https://doi.org/10.1007/ s11136-020-02555-w

33. Graham, I. D., Logan, J., Harrison, M. B., Straus, S. E., Tetroe, J., Caswell, W., et al. (2006). Lost in knowledge translation: time for a map? The Journal of Continuing Education in the Health Professions, 26(1), 13-24. https://doi.org/10.1002/chp.47

34. Wilson, I. B., \& Cleary, P. D. (1995). Linking clinical variables with health-related quality of life. A conceptual model of patient outcomes. JAMA, 273(1), 59-65.

35. Samuel, C. A., Smith, A. B., Elkins, W., Richmond, J., Mahbooba, Z., Basch, E., et al. (2020). Racial differences in user experiences and perceived value of electronic symptom monitoring in a cohort of black and white bladder and prostate cancer patients. Quality of Life Research. https://doi.org/10.1007/s11136-020-02442-4

36. Richards, H. S., Portal, A., Absolom, K., Blazeby, J. M., Velikova, G., \& Avery, K. N. L. (2020). Patient experiences of an electronic 
PRO tailored feedback system for symptom management following upper gastrointestinal cancer surgery. Quality of Life Research. https://doi.org/10.1007/s11136-020-02539-w

37. Naughton, M. J., Salani, R., Peng, J., Lustberg, M., DeGraffinreid, C., Moon, J., et al. (2020). Feasibility of implementing a text-based symptom-monitoring program of endometrial, ovarian, and breast cancer patients during treatment. Quality of Life Research. https://doi.org/10.1007/s11136-020-02660-w

38. Mouillet, G., Falcoz, A., Fritzsch, J., Almotlak, H., Jacoulet, P., Pivot, X., et al. (2021). Feasibility of health-related quality of life (HRQoL) assessment for cancer patients using electronic patient-reported outcome (ePRO) in daily clinical practice. Quality of Life Research. https://doi.org/10.1007/ s11136-020-02721-0

39. Teela, L., van Muilekom, M. M., Kooij, L. H., Gathier, A. W., van Goudoever, J. B., Grootenhuis, M. A., et al. (2020). Clinicians' perspective on the implemented KLIK PROM portal in clinical practice. Quality of Life Research. https://doi.org/10.1007/ s11136-020-02522-5

40. Braunschneider, L. E., Lehmann, M., Magaard, J. L., Seeralan, T., Marx, G., Eisele, M., et al. (2020). GPs' views on the use of depression screening and GP-targeted feedback: a qualitative study. Quality of Life Research. https://doi.org/10.1007/ s11136-020-02703-2

41. Boyce, M. B., Browne, J. P., \& Greenhalgh, J. (2014). The experiences of professionals with using information from patientreported outcome measures to improve the quality of healthcare: a systematic review of qualitative research. BMJ Quality and Safety, 23(6), 508-518. https://doi.org/10.1136/bmjqs-2013-002524

42. Schilling, V., Zimmermann, D., Rubel, J. A., Boyle, K. S., \& Lutz, W. (2020) Why do patients go off track? Examining potential influencing factors for being at risk of psychotherapy treatment failure. Quality of Life Research. https://doi.org/10.1007/ s11136-020-02664-6.

43. Lutz, W., Rubel, J. A., Schwartz, B., Schilling, V., \& Deisenhofer, A. K. (2019). Towards integrating personalized feedback research into clinical practice: development of the trier treatment navigator (TTN). Behaviour Research and Therapy, 120, 103438. https:// doi.org/10.1016/j.brat.2019.103438

44. Spitzer, W. O. (1987). State of science 1986: quality of life and functional status as target variables for research. Journal of Chronic Diseases, 40(6), 465-471.

45. Lutz, W. (2002). Patient-focused psychotherapy research and individual treatment progress as scientific groundwork for an empirical based clinical practice. Psychotherapy Research, 12, 251-273.

46. Espallargues, M., Valderas, J. M., \& Alonso, J. (2000). Provision of feedback on perceived health status to health care professionals: a systematic review of its impact. Medical Care, 38(2), 175-186. https://doi.org/10.1097/00005650-200002000-00007

47. Valderas, J., Kotzeva, A., Espallargues, M., Guyatt, G., Ferrans, C., Halyard, M., et al. (2008). The impact of measuring patientreported outcomes in clinical practice: a systematic review of the literature. Quality of Life Research, 17(2), 179-193. https://doi. org/10.1007/s11136-007-9295-0

48. Greenhalgh, J., \& Meadows, K. (1999). The effectiveness of the use of patient-based measures of health in routine practice in improving the process and outcomes of patient care: a literature review. Journal of Evaluation in Clinical Practice, 5(4), 401-416. https://doi.org/10.1046/j.1365-2753.1999.00209.x

49. Greenhalgh, J. (2008). The applications of PROs in clinical practice: what are they, do they work, and why? Quality of Life Research, 18(1),115-123. https://doi.org/10.1007/ s11136-008-9430-6

50. Kendrick, T., El-Gohary, M., Stuart, B., Gilbody, S., Churchill, R., Aiken, L., et al. (2016). Routine use of patient reported outcome measures (PROMs) for improving treatment of common mental health disorders in adults. Cochrane Database Syst Rev, 7, CD011119, doi:https://doi.org/10.1002/14651858.CD011119. pub2

51. Boyce, M. B., \& Browne, J. P. (2013). Does providing feedback on patient-reported outcomes to healthcare professionals result in better outcomes for patients? A systematic review. Quality of Life Research, 22(9), 2265-2278. https://doi.org/10.1007/ s11136-013-0390-0.

52. Ishaque, S., Karnon, J., Chen, G., Nair, R., \& Salter, A. B. (2019). A systematic review of randomised controlled trials evaluating the use of patient-reported outcome measures (PROMs). Quality of Life Research, 28(3), 567-592. https://doi.org/10.1007/ s11136-018-2016-z

53. Mackrill, T., \& Sørensen, K. M. (2019). Implementing routine outcome measurement in psychosocial interventions - a systematic review. European Journal of Social Work, 23(5), 790-808. https://doi.org/10.1080/13691457.2019.1602029

54. Campbell, R. D., \& Rutherford, C. (2021). Perceived benefits and limitations of using patient-reported outcome measures in clinical practice with individual patients: a systematic review of qualitative studies. Quality of Life Research. https://doi.org/10.1007/ s11136-021-03003-z

55. Ovretveit, J., Zubkoff, L., Nelson, E. C., Frampton, S., Knudsen, J. L., \& Zimlichman, E. (2017). Using patient-reported outcome measurement to improve patient care. International Journal for Quality in Health Care, 29(6), 874-879. https://doi.org/10.1093/ intqhe/mzx 108

56. Greenhalgh, J., Long, A. F., \& Flynn, R. (2005). The use of patient reported outcome measures in routine clinical practice: lack of impact or lack of theory? Social Science \& Medicine, 60(4), 833-843. https://doi.org/10.1016/j.socscimed.2004.06.022

57. Greenhalgh, J., Dalkin, S., Gooding, K., Gibbons, E., Wright, J., Meads, D., et al. (2017). Functionality and feedback: a realist synthesis of the collation, interpretation and utilisation of patientreported outcome measures data to improve patient care. Health Services and Delivery Research, 5(2), 1-280. https://doi.org/10. 3310/hsdr05020

58. Austen, J. (1813). [1998]). Pride and Prejudice. Project Gutenberg. Accessed 22.09.2021.

59. Jensen-Doss, A., Douglas, S., Phillips, D. A., Gencdur, O., Zalman, A., \& Gomez, N. E. (2020). Measurement-based care as a practice improvement tool: Clinical and organizational applications in youth mental health. Evidence-Based Practice in Child and Adolescent Mental Health, 5(3), 233-250. https://doi.org/10. 1080/23794925.2020.1784062.

60. Black, N., Burke, L., Forrest, C. B., Sieberer, U. H., Ahmed, S., Valderas, J. M., et al. (2016). Patient-reported outcomes: pathways to better health, better services, and better societies. Quality of Life Research, 25(5), 1103-1112. https://doi.org/10.1007/ s11136-015-1168-3

61. Porter, I., Goncalves-Bradley, D., Ricci-Cabello, I., Gibbons, C., Gangannagaripalli, J., Fitzpatrick, R., et al. (2016). Framework and guidance for implementing patient-reported outcomes in clinical practice: evidence, challenges and opportunities. Journal of Comparative Effectiveness Research, 5(5), 507-519. https://doi. org/10.2217/cer-2015-0014.

62. Kish, L. J., \& Topol, E. J. (2015). Unpatients-why patients should own their medical data. Nature Biotechnology, 33(9), 921-924. https://doi.org/10.1038/nbt.3340

63. Cruz Rivera, S., Mercieca-Bebber, R., Aiyegbusi, O. L., Scott, J., Hunn, A., Fernandez, C., et al. (2021). The need for ethical guidance for the use of patient-reported outcomes in research and clinical practice. Nature Medicine, 27(4), 572-573. https://doi. org/10.1038/s41591-021-01275-Z

64. Muir, H. J., Coyne, A. E., Morrison, N. R., Boswell, J. F., \& Constantino, M. J. (2019). Ethical implications of routine outcomes 
monitoring for patients, psychotherapists, and mental health care systems. Psychotherapy (Chicago, Ill.), 56(4), 459-469. https:// doi.org/10.1037/pst0000246

65. Nguyen, H., Butow, P., Dhillon, H., \& Sundaresan, P. (2021). A review of the barriers to using patient-reported outcomes (PROs) and patient-reported outcome measures (PROMs) in routine cancer care. Journal of Medical Radiation Sciences, 68(2), 186-195. https://doi.org/10.1002/jmrs.421.

66. Hancock, S. L., Ryan, O. F., Marion, V., Kramer, S., Kelly, P., Breen, S., et al. (2020). Feedback of patient-reported outcomes to healthcare professionals for comparing health service performance: a scoping review. British Medical Journal Open, 10(11), e038190. https://doi.org/10.1136/bmjopen-2020-038190

Publisher's Note Springer Nature remains neutral with regard to jurisdictional claims in published maps and institutional affiliations. 